\author{
Gostracts.
}

\title{
PHARYNX AND NASO-PHARYNX.
}

Kowler (Mentone)--Retro-pharyngeal Abscess; Operation; Sudden Death._. Ann. des Mal. de l'Oreille, etc.," vol. xxxviii, Part XII.

A child, aged two, who had suffered three weeks previously from influenza, developed respiratory trouble; swallowing had been impossible for four days, and milk regurgitated by the nose. The voice was nasal in character and breathing became more and more difficult, accompanied by stridor and prominence of the eyes. The right side of the neck was swollen, and the cervical glands were enlarged. Oral examination revealed a bulging in the right tonsillar region extending over to the left side of the middle line. On palpation the swelling felt tense, its upper pole could be defined, but the lower, which extended deeply, could not be reached. In view of the danger which might arise from the sudden rupture of so large an abscess, the author decided to partially evacuate it. This was effected by introducing a blunt hook into the upper part of the swelling. Pus flowed, and palpation afterwards gave the impression of feeling a collapsed ball. The following day the abscess-cavity had refilled. The child was then placed on the knees of an assistant with its head inclined downwards and forwards and an opening made with a blunt hook into the lower part of the swelling. A flood of pus filled the mouth and spurted on to the assistant's feet. As soon as the discharge ceased, the child suddenly turned her head backwards, became deathly pale and expired. The author remarks that all the methods up to the present vaunted, may fail to avoid these catastrophes, and wonders whether gradual emptying of these extensive abscesses by aspiration would not prevent them.

The technique would be as follows: (1) To lightly anæsthetise the patient. (2) To adopt Rose's position. (3) To puncture with a trocar and partially evacuate the abscess. (4) Palpation of the pharynx to gauge the condition of the abscess. (5) Only to have recourse to a second puncture twelve hours after the preceding, till the abscess is evacuated.

H. Clayton Fox.

\section{Robertson, W. N.-Recurring Fibroma of the Naso-pharynx. "Austra-} lasian Medical Gazette," June 7, 1913.

A boy, aged ten, was operated on in 1911 for a dense fibrous growth which projected under the zygoma, right side, in front of the masseter muscle. The growth was removed by external incision. It continued through the pterygo-maxillary fossa, and the final portion, which was removed by traction, like a cork out of a bottle, was supposed to come from the sphenoidal sinus of the opposite side. No growth was noticed then in the naso-pharynx. Patient recovered. Eighteen months later he came again. There was a growth in right nostril, and a large mass in naso-pharynx. Preliminary trachectomy ; splitting of soft palate, enucleation of growth with elevators. A prolongation through pterygo-maxillary fissure could not be enucleated. It was seized with a pair of necrosis forceps and twisted out. Recovery. A. J. Brady. 


\section{NOSE.}

Heermann (Essen).--Resection of the Septum in Childhood, and the Prevention of Perforation and Flapping. "Zeitschr. f. Laryngol.," Bd. vi, Heft 2.

The writer comes to the following conclusions: The submucous resection of the septum, if not too extensive, can be satisfactorily carried out in children with the aid of local anæsthesia alone. It does not lead to post-operative disturbance of the growth of the nose.

Septal spines also should be removed by the submucous method, and not by the saw.

J. S. Fraser.

\section{Schulz, Adolf (Dantzig).-.-Local Anæsthesia for the Submucous Re- section. "Zeitschr. f. Laryngol.," Bd. vi, Heft 3.}

Schulz dissolves a few grains of alypin in suprarenin in a saucer, and applies this on a cotton tampon. After fifteen minutes good anæsthesia and bloodlessness are obtained. He uses the same method for the intranasal operations on the antrum, and also for opening the sphenoidal sinus. He finds the mixture less toxic than cocaine with suprarenin.

$$
\text { J. S. Fraser. }
$$

Renner, W. Scott.-The Middle Turbinated Body : Some Indications for its Removal. "Annals of Otology, etc.," xxi, p. 443.

Does not refer so much to removal as a part of more radical operations, but rather in connection with reflex neuroses and to assist drainage of accessory sinuses.

Macleod Yearsley.

\section{Cobb, F. K.-Atrophic Rhinitis and its Treatment by Vaccines.} “Annals of Otology, ete.," xxi, p. 463.

In ninety cases cultures were taken after sterilisation of the vestibule. All showed a pure culture of the atrophic bacillus. Forty patients received vaccines, from four to fifty-six injections. The author considers that vaccine injections, if carried out regularly, seem to produce permanent improvements. Irregular or small number of injections cannot be counted on with the same certainty, but marked improvement seems to have followed, even in these cases. As to strength of vaccine, each cubic centimetre contains 100 million bacteria, and the dose varied from $\frac{1}{10} \mathrm{c.cm}$. to $1 \frac{1}{2} \mathrm{c.cm}$.

Macleod Yearsley.

Polyák, L. (Budapest).-The Technique of Intra-nasal Dacryocystostomy. "Archiv für Laryngol.," vol. xxvii, Part III.

A considerable advance was made in the treatment of obstruction of the nasal duct when Toti, in 1904, introduced his method of dacrocystorhinostomy. By means of an external operation the inner (nasai) mucoperiosteal and bony walls of the lachrymal sac are resected so as to secure a wide communication between the sac and the nasal cavity. The lachrymal sac, apart from the portion resected, is preserved and the canaliculi are not slit. In the years 1909 and 1910 both the author and West, independently of one another, devised their methods of windowresection of the duct in the middle meatus without interference with the turbinals. These intra-nasal operations on the duct proved satisfactory in a number of cases, but failed in others in which the stricture was high up or the sac itself much diseased. In such cases the external operation of Toti on the sac was still required. The author has now elaborated a method embodying all the prineiples of Toti's operation, but performed 
through the nose. The duct is opened by means of a chisel immediately in front of the anterior end of the middle turbinal. One blade of a specially constructed cutting-forceps is then inserted and pushed upwards, the jaws of the instrument gradually cutting away the whole of the membranous and bony wall between the sac and the nose. During this process the anterior point of attachment of the middle turbinal is removed, and frequently a few of the ethmoidal cells are opened, as is also the case in the operation of Toti, all the requirements of which are fulfilled without the disadvantage of an external scar. Thomas Guthrie.

Baumgarten (Budapest).-Cure and Improvement of Visual Disturbances by Nasal 0peration. “Archiv. f. Laryngol.," vol. xxv, Part I.

This paper adds another six cases to the eleven already reported by the author in the Monatschrift für. Ohrenheilkunde, 1910, No. 9, and 1911, No. 6. Of the total number of seventeen cases, fourteen were completely or almost completely cured of their visual disturbance or blindness, in two marked improvement took place, and in one moderate improvement. A similar result is to be expected in all acute and even chronic cases in which the oculist definitely attributes the eye affection to a nasal cause. Of the seventeen cases, five were classed as retrobulbar neuritis, two as amblyopia with normal fundus, three as papillitis acuta, two as papillitis chronica ; in three cases neuritis acuta was found, and in two decoloratio papilæ. Central scotoma was present in five cases, paracentral in one, and central colour scotoma in eight. The colour scotoma disappeared in all cases immediately after the first operation, and was always the first sign of improvement.

In reference to the nasal conditions found in these cases and the treatment required the author recognises three groups:

(1) Accessory sinus disease is present, and must receive immediate and energetic treatment.

(2) There is a large ethmoidal bulla or marked swelling of the middle turbinal, which should always be at once removed.

(3) The middle turbinal is only slightly thickened, or is normal and in contact with the septum. Even in such cases, when the oculist is positive as to the nasal origin of the trouble, operative measures are indicated. Application of cocaine and adrenalin solution to the middle turbinal will sometimes result in an immediate improvement of the vision, but the absence of such improvement should not be regarded as contra-indicating operation. In all these cases the author removes at once enough of the middle turbinal to expose the anterior wall of the sphenoidal sinus, which can then easily be opened if required on the following day.

The most marked visual disturbance is usually associated with accessory sinus disease. In other cases the amount of visual disturbance bears little relation to the degree of enlargement of the bulla or middle turbinal. Under these circumstances the statement of the oculist that the trouble is of nasal origin must suffice, and any delay in resort to operation may be of serious danger to the eye.

Thomas Guthrie.

Levinstein, Dr. 0swald (Berlin).-The Pathology and Treatment of the Nasal Reflex Neuroses which proceed from the Tubercle of the Septum. " Zeitschr. f. Laryngol.," Bd. vi, Heft 2.

The first case recorded in this paper is that of a female, aged eighteen, who complained of sudden redness of the left eye, with lachrymation and 
pain if she strained her sight at all; these attacks lasted from five to ten minutes, and were accompanied by tickling in the nose and pain between the nose and eye. On examination the septal tubercle on the left side was very sensitive. Pain in the left eye, lachrymation and injection of the conjunctiva were produced when this part was touched with a probe, but these symptoms at once passed off on the application of cocaine. Levinstein cauterised the tubercle on three occasions and obtained a cure.

The second case is that of a female, aged twenty, who had suffered from attacks of frontal headache for one year. In this case also the nose was normal except for hyperæsthesia of the septal tubercle. The cocaine experiment was again positive, and deep application of the cautery on both sides resulted in cure.

Six similar cases are reported, but in two the tubercle of the septum was hyperplastic. Levinstein thinks that the condition present in these cases was due to a sudden filling with blood of the cavernous spaces in the tubercle of the septum.

J. S. Fraser.

Coolidge, A,-The Etiology of Hay-Fever. "Boston Med. and Surg. Journ.," April, 1913.

Smith, T. (Boston).--The Etiology of Hay-Fever. “ Boston Med. and Surg. Journ.," April, 1913.

These two papers both review the more recent theories of the causation of the condition. Coolidge criticises the view advocated by many observers that the primary cause may be found in an instability of the local nerves. Septal deviations or other intra-nasal deformities and the uric acid diathesis are not more satisfactory as ætiological factors. The opinion he is most in favour of is that the primary cause is a biochemical reaction. Both authors refer to the work of Dunbar, who maintained that the exciting agent was a soluble toxin, and therefore must be rendered inert if an antitoxin were prepared and injected into sensitive individuals. By treatment with the serum which he prepared, Dunbar claimed 60 per cent. of cures. In recent years many observers have been struck with the similarity of the phenomena of hay-fever and that of an anaphylactic reaction. This is in direct antagonism to Dunbar's claim, but the rapidity of onset, the asthmatic symptoms and the incapacity to become immunised are in favour of anaphylaxis. Reference is made to Neisser's work. He has recently attempted to secure active immunity by giving increasing doses of pollen toxin; only some fifteen cases were treated, and it is claimed that half the number were benefited. $\quad J . A$. Knowles Renshaw.

\section{LARYNX.}

Menier, Dr. Marius (Decazeville, France).-The History of the Treatment of Laryngeal Tuberculosis. "Zeitschr. f. Laryngol.," Bd. vi, Heft 2.

Dr. Menier points out that voice rest dates back to 1838 and was introduced by Colombat, while tracheotomy in the treatment of phthisis laryngea was first described by Carmichael in 1833 in the Dublin Medical and Surgical Journal.

J. S. Fraser.

Levy, Robert.--Laryngeal Tuberculosis. “Journ. Amer. Med. Assoc.," May 17, 1913.

In reviewing the subject of laryngeal tuberculosis the author considers it of such frequent occurrence as to warrant the examination of the 
upper air-passages of all cases, or suspected cases, of pulmonary tuberculosis. During the first or earliest manifestation of laryngeal involvement are a slight intermittent hoarseness, excessive sensitiveness and irritability of the pharynx, painful sensations in throat, marked pallor of mucous membrane, of pharynx and larynx, limited movements, slight redness and swelling of the corresponding vocal cord. The onset of tuberculous deposit in the submucous tissue is frequently marked by early pain, and the development of ulceration is characterised by increase in the pain.

Generally speaking the advent of laryngeal complication adds to the gravity of the prognosis. In the matter of treatment an early diagnosis is of paramount importance, and Levy groups the various therapeutic measures under the following heads:

(1) Treatment by means of rest to the vocal organ in conjunction with rest and general hygienic measures.

(2) Local measures-modifications of surgical procedures. Among them the use of the galvano cautery seems to have the most advocates.

(3) The relief of pain by injection or section of the superior laryngeal nerve.

(4) The use of tuberculin.

The most beneficial effect of rest is seen in the cases with unilateral redness or moderate infiltration, but even the ulcerative cases, if not too far advanced, are also markedly benefited. All obstructive lesions of the nose should be appropriately dealt with in order to establish a more healthy condition of the upper air-passages, thereby preventing a chronic catarrhal laryngitis, which, once established, predisposes to tuberculous laryngeal complication. Fresh air, an abundance of sunshine and rest, and a maximum of nutritious food are as essential for the local as for the general condition.

The galvano-cautery may be used in all forms of the disease. Superficial ulcers heal readily under its action, while odematous infiltrative swellings frequently shrink to comparatively small size. Other more extensive surgical measures may be carried out to advantage in many cases, depending on the extent of the lesion and its limitations.

For the relief of intolerable pain, section or injection with alcohol of the superior laryngeal nerve is advised, preference being given to the latter procedure owing to its simplicity and satisfactory result.

The use of tuberculin must be considered only as an adjunct to local treatment, and its administration must be carefully conducted, preferably in sanatoria.

Birkett (Rogers).

\section{EAR.}

Kabatschnik, M.-A $\mathbf{N} \in \mathbf{W}$ Hearing Test. “Monats. f. Ohrenh.," Year 46 , No. 11.

The very considerable variations in the structural consistency of the mastoid process must, in the opinion of the author, so seriously prejudice the accuracy of those tests associated with the names of Rinne and Schwabach that he has been led to introduce other methods, which, he claims, eliminate these errors.

His test consists essentially in occluding the external auditory meatus of the ear in question with a finger, to the nail of which the handle of a vibrating tuning-fork is then applied. The inference is drawn in the usual way by noting the report of the patient as to the intensity of the sound in this position compared with that when the fork is held 
before the unoccluded ear. Differential diagnosis between affections of the sound-conducting apparatus and sensation is made in a corresponding manner to that adopted in the original tests.

In addition to an improvement on the older methods the author considers that with his modification bone-conduction from the affected side to the healthy ear is "scarcely possible," and further, that it may prove useful in detecting malingering.

[It is difficult to understand how the writer can have overlooked the fact that occlusion of the meatus at once introduces the possibility of the production of those phenomena on which Gellés test is based, and which thus surely renders his proposed modifications unreliable in this respect -it is obvious that a difference in the force with which the meatus is occluded may easily lead to a varying report in the case of a functional stapedio-vestibular articulation.-A. R. T.]

Alex. R. Tweedie.

Denker, Prof. Alfred.- Upon the Present Status of Otosclerosis. "Annals of Otology," xxii, p. 1.

In this admirable paper Prof. Denker defines otosclerosis as a disease in which there is a permeable tube and a normal tympanic membrane, accompanied by a definite and characteristically marked clinical picture of a progressive difficulty in hearing, as shown by functional tests and a pathologico-anatomical picture showing loss of movement of the stapes from bony ankylosis and progressive spongification of the bony labyrinthine capsule, with, in addition, an atrophic degenerative process in the nerve-endings in the membranous labyrinth. He prefers to retain the old title of "otosclerosis" until its ætiology and pathological anatomy are cleared up. He discusses the opposed theories as to the primary or secondary nature of the condition, and corrects the erroneous statement of Heimann and Fröschels that he (Denker) considered it as following middle-ear inflammation. He points out that the coincidence of pregnancy with the beginning of otosclerosis suggests a causal relationship between the function of the hypophysis cerebri and the ear disease, and surmises that there is a possibility of an rtiological connection between malfunction of the hypophysis and the origin of otosclerosis, especially in persons with an hereditary taint, acknowledging, however, that only a portion of the cases can be thus explained. From the variability of the lesions found and the resulting symptom-complex, otosclerosis may be divided into three forms: (1) Isolated stapes ankylosis; (2) multiple spongifying foci in the labyrinth capsule, with atrophic degeneration of the membranous labyrinth without stapes ankylosis; (3) a combination of (1) and (2). Discussing the subject of treatment, Denker is against local operative treatment. Otomassage is useful when subjective noises are marked. He thinks more is to be expected from the internal administration of phosphorus than from iodide of potassium, diplococcus serum, etc. He has used phosphorus for many years - for the past three in the form of phytin. Recently he has been giving hypophysin or pituitrin. In women, ovarian or spermin tablets may be tried. Of otosclerol and vasotonin he has no experience.

Macleod Yearsley.

Reh and Méroz.-Pseudo-diphtheria Bacillus in Cases of Otitis Media. “ Rev. Med. de la Suisse Romande," January, 1913.

Pseudo-diphtheria bacilli are often present in the pus in otitis media, generally associated with strepto-, staphylo-, or pneumococi, rarely in pure culture. They come from the naso-pharynx or from the external 
meatus, and have no pathogenic significance. For this reason it is important to have some ready means of distinguishing them from true Loeffler's bacilli. The authors consider that hitherto the only sure method for making such differential diagnosis was by inoculation of guinea-pigs, as their morphological, cultural, staining characters, etc., are either inconstant, or require too prolonged investigation to be of any clinical value. The authors have devised a method of making this differential diagnosis. It depends on the fact that Loeffler's bacillus when stained by Gram's method retains the staining only on condition of not being exposed to the action of alcohol too long, whereas pseudodiphtheria bacillus retains the staining even when exposed to the action of alcohol for an indefinite time. Their method has not been used in many cases, but so far has given positive results.

\section{Arthur J. Hutchison.}

Gatscher, Siegfried.-Fatal Mediastinitis, the Result of a Retropharyngeal Abscess following Acute Purulent Otitis Media. “Monatss. f. Ohrenh.," year 47, No. 5.

A man, aged thirty-three, was brought into Prof. Urbantschitsch's clinic in a quite neglected condition, with the report that his left ear had been affected for the last five weeks, following an attack of influenza. Some days after the trouble commenced he had pain on swallowing, which, however, soon ceased. There was now a profuse fotid purulent discharge from the left ear, the tympanic membrane of which was injected and hard to see on account of the secretion. No tenderness of the mastoid. Two days later pressure on the left side of the throat caused discharge of pus from the meatus, the left tonsil was slightly swollen, the anterior auricular glands enlarged, and the antrum tender.

Operation revealed a diseased antrum and cells extending throughout the whole bone from the tegmen to the tip. The next day, as on examination of the pharynx a profuse flow of pus appeared in and around the left linguo-tonsillar region, the patient was transferred to the laryngological clinic. Here, as his condition became so much worse, the mediastinum was opened on both sides, but the patient rapidly succumbed.

Post-mortem.-Pus had apparently broken through the floor of the tympanum and tracked down and around the pharynx and larynx, passing over to the opposite side, and eventually infecting the mediastinum on both sides.

Alex. R. Tweedie.

Tovey (Jun.), G. L.-Operative Treatment of Sinus Thrombosis. "Annals of Otol., Rhinol., and Laryngol.," vol. xxi, p. 362.

A useful paper, giving a summary of 73 cases (48 males, 25 females), which is, briefly, as follows: Right ear, 49 ; left ear, 24 ; acute, 56 ; chronic, 17. Temperature: septic, 60 ; variable, with remissions, 9; none, 4. Chills : distinct, 41 ; chilly sensation, 19 ; none, 13 . Eyes: optic neuritis, 12 ; slight engorgement, 7 ; no change, 54 . Blood : white

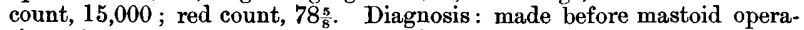
tion, 32 ; secondary to mastoid operation, 30 ; at time of mastoid operation, 11 (sinus wall necrosed in 3). Metastases : 6 cases after ligature of vein. Result: well, 61 ; died, 12 (cavernous sinus thrombosis, 2 ; general septicæmia, with metastases, 4 ; admitted unconscious and in extremis, 5; scarlatina intercurring during convalescence, 1). Sinus thrombosis found as complication in following fatal cases: tuberculous 
meningitis, 1; septic meningitis, 6; temporo-sphenoidal abscess, 3; cerebellar abscess, 1 .

Macleod Yearsley.

\section{MISCELLANEOUS.}

Marshall.-Correction of External Nasal Deformities. "The Journal of the American Medical Association," January 18, 1913.

For the correction of external deformities of the nose, most of which are combined with serious nasal obstructions, the author has devised a new operation, the essential feature of which is to cut through the nasal process of the superior maxillary bone. The technique of the operation is as follows: With a narrow-bladed knife an incision is made directly over the nasal process of the superior maxillary bone at the point where the elevation which makes the nasal prominence begins. The incision is made parallel to the normal line of the nose, a length of $0.25 \mathrm{~cm}$. The nasal process is cut through at this point with a bevelled chisel, and pressure is thus made by an assistant to control bleeding, while the opposite side is treated in like manner. By means of a broad-bladed forceps, one blade of which enters the nasal passage, the opposite blade remaining outside, the operator completes by a fracture the mobility of the nasal process along its entire line. Both sides are similarly dealt with. If there is a nasal obstruction through the malposition of the septum, the septum is seized with the same forceps and forced into a correct position by loosening its articulations without separating them. The nose is not likely yet to be in a straight line, the defect lying at the suture between the frontal and upper extremities of the two nasal bones and both processes of the superior maxilla. The faulty angle can be straightened by a sharp stroke with the mallet at this point, guarded by a rubber-covered lead plate, the force being directed downwards from the frontal bone, but toward the obtuse angle. Elevation can be assisted sometimes to advantage with a large urethral sound.

The lines of incision are covered by iodoform gauze and collodion; the lower part of the nose is encased in a collodion dressing, and in some cases nasal splints are inserted for twenty-four hours or more.

Birkett (Rogers).

\section{REVIEWS.}

Asthma and its Radical Treatment. By Jas. ADAM, M.D., F.R.F.P.S. Pp. 184. Illustrated. London: Henry Kimpton, 1913. Price 5s.

The author treats his subject under eleven headings, and of these not the least interesting are his introductory remarks, wherein be condemns the labelling of asthma as a neurosis, and formulates his thesis, viz. that asthma is primarily a toxæmia. He brings evidence that arterial tension is low rather than high in asthmatics, even during " an attack," and cites the periodicity of asthma, the urine conditions, as well as the results he has obtained from dietetic treatment to support his view that asthma is a toxæmia. Rhinologists will be interested in the sections where the author directs attention to nasal abnormalities in connection with asthma. He lays stress on the importance of removing adverse nasal conditions, but equally emphasises the importance of having regard to the essential underlying cause of asthna, viz. the toxæmic state, and regards 